\title{
Correlated time-varying magnetic fields and the core size of Mercury
}

\author{
I. Wardinski ${ }^{1}$, B. Langlais ${ }^{1} \&$ E. Thébault ${ }^{1}$ \\ ${ }^{1}$ Laboratoire de Planétologie et Géodynamique, Université de Nantes, Université d'Angers, CNRS, UMR \\ 6112, Nantes, France
}

\section{Key Points:}

- We model Mercury's magnetic field on a temporal basis with spherical harmonics

- We analyze time varying external (inducing) and internal (induced) magnetic fields

- We estimate Mercury's core size at $2060 \pm 22 \mathrm{~km}$

Corresponding author: I. Wardinski, ingo.wardinski@univ-nantes.fr 


\begin{abstract}
Mercury is characterized by a very peculiar magnetic field, as it was revealed by the MESSENGER mission. Its internal component is highly axisymmetric, dominated by the dipole, and very weak. This in turns leads to a very dynamic magnetosphere. It is known that there exist relationships between the internally generated field and the external field, although their dynamics are complex. In this study we derive steady and time-varying spherical harmonic models of Mercury's magnetic field using MESSENGER measurements, and interpret these models both in terms of correlated features and of the internal structure of Mercury. The influence of the hemispheric data distribution of MESSENGER is evaluated to grant the robustness of our models. We find a quadrupole-to-dipole ratio of 0.27 for the steady magnetic field. The time-varying models reveal periodic and highly correlated temporal variations of internal and external origins. This argues for externally inducing and internally induced sources. The main period is 88 days, the orbital period of Mercury around the Sun. There is no measurable time lag between variations of external and internal magnetic fields, which place an upper limit of $1 \mathrm{Sm}^{-1}$ for the mantle conductivity. Finally, the compared amplitudes of external and internal time varying field lead to an independent (from gravity studies) estimate of the conductive core radius, at $2060 \pm 22 \mathrm{~km}$. These analyses will be further completed with the upcoming BepiColombo mission and its magnetic field experiment, but the presented results already lift the veil on some of the magnetic oddities at Mercury.
\end{abstract}

\title{
1 Introduction
}

Since the beginning of its exploration with space-borne missions, it is known that Mercury has a magnetic field of internal origin (Ness et al., 1974a). This internal magnetic field is relatively weak, $\sim 1 \%$ of Earth's magnetic field strength. It is characterized by a strong axisymmetry and a large quadrupole-to-dipole ratio (Anderson et al., 2012; Johnson et al., 2012; Oliveira et al., 2015; Thébault et al., 2018). This internal field is significantly larger than the interplanetary magnetic field, and its interaction with the solar wind forms a bow shock wave and a magnetosphere (Ness et al., 1974b).

Several mechanisms have been proposed that could generate Mercury's weak internal magnetic field. These incorporate a thermo-electric dynamo process at a topographically rough outer core surface (Stevenson, 1987), a dynamo driven by a thermo-compositional convection associated with the solidification of an inner core (Christensen, 2006), or a 
dynamo process driven by convection that is affected by a radial gradient of the electrical conductivity at the outer core surface (Gómez-Pérez et al., 2010). Alternatively, Aharonson et al. (2004) suggested that the internal magnetic field could also be generated by the magnetization of Mercury's crust and mantle due to an ancient (and perhaps extinct) dynamo process.

A number of studies highlighted the importance of the magnetospheric magnetic field, which may control the internal magnetic field generation of Mercury by a feedback mechanism (Glassmeier et al., 2007; Gómez-Pérez \& Solomon, 2010; Heyner et al., 2011). The magnetosphere of Mercury results from an interaction of the solar wind and the planetary magnetic field. Its subsolar stand-off distance is $0.45 R_{M}$ above the surface of the planet, where $R_{M}=2440 \mathrm{~km}$ (Johnson et al., 2012; Winslow et al., 2013; Thébault et al., 2018). This interaction causes an electrical current to flow on the boundary of the magnetosphere across the tail of the magnetosphere, similar to the Chapman-Ferraro current system in Earth's magnetosphere (Chapman \& Ferraro, 1940, 1941).

Mercury's orbital motion leads to periodic variations of the solar wind conditions that cause varying stand-off distances of the magnetosphere and variations of the magnetospheric magnetic field (Suess \& Goldstein, 1979). One peculiarity of the Hermean system is related to the 3:2 resonance between the rotation of Mercury and its revolution around the Sun. It takes 3 rotations of the planet (58.65 days each), or 2 full orbits (87.67 days each), for Mercury to return to similar solar conditions, i.e. a given location sunlit under the same angle. The synodic period, i.e. the rotation of the Sun as seen from Mercury as it moves along its orbit, is close to 36 days.

As mentioned above, variations of the magnetospheric stand-off distance cause varying Chapman-Ferraro currents and generate a time-varying external magnetic field, which in turn induces a time-varying internal magnetic field in Mercury's electrically highly conducting core (Hood \& Schubert, 1979; Glassmeier, 2000; Grosser et al., 2004; Johnson et al., 2016). The induced magnetic field adds to the primary internal magnetic field. Several studies (Glassmeier, 2000; Grosser et al., 2004) estimated the induced magnetic field amplitude may reach about $10 \%$ of the mean internal magnetic field intensity at the planet's surface. Closely related to magnetic field generation and the induction process is Mercury's internal structure. Earth-based observations confirmed the presence of a metallic core, that contains a liquid part inside (Margot et al., 2007). The core of Mercury is 
covered by a mantle, which may resemble Earth's mantle in physical properties such as composition and electrical conductivity (Rivoldini et al., 2009; Zhang \& Pommier, 2017). Geodetic observation of Mercury's gravity field during the MESSENGER mission confines the core radius to $2004 \pm 39 \mathrm{~km}$ (Rivoldini \& Van Hoolst, 2013); in combination with Earth-based radar observations of the planet's spin state the core size can be estimated as $2020 \pm 30 \mathrm{~km}$ (Hauck et al., 2013). Note that these two results are estimates of the liquid core radius. As pointed out by Hauck et al. (2013), the uppermost part of the core could be solid and indistinguishable from the mantle. Johnson et al. (2016) found Mercury's core based on induction analyses at 1900-2200 km. The latter study highlighted the potential of MESSENGER's magnetic field measurements to infer the internal structure of Mercury. In this case, the radius estimate is that of the electrically conductive core, regardless of its solid or liquid state.

The rapid dynamics of the solar wind, associated with the weak magnetic field of Mercury and its orbital motion, lead to both fast magnetospheric changes and slow periodic variations. The aim of this study is thus to analyze and to compare the temporal variability of external and internal constituents of Mercury's magnetic field. Analyses are based on magnetic field measurements made by NASA's MESSENGER mission (Solomon et al., 2007). The paper is organized as follows. First we describe the used methods to isolate the static, or steady, constituents of the internal and external magnetic fields of Mercury. We next derive residuals between the steady field model and magnetic field measurements of MESSENGER, and we model them with a time-varying scheme in the third section. Results are analyzed in the fourth section, where coherency between internal and external field variations is investigated. This allows to specify possible mechanisms that may generate and drive these magnetic field variations. One important output is a new and non-geodetic estimate of the conductive core radius. We conclude our study in the last section.

\section{Derivation of a steady magnetic field model}

\subsection{Data selection}

The MESSENGER spacecraft remained in orbit around Mercury from 18 March 2011 and lasted until 30 April 2015. During this period of 4 years, the spacecraft continuously measured Mercury's magnetic field. The mission orbit was highly elliptical, with 
a periapsis ranging from 200 to $500 \mathrm{~km}$ over the north polar region, and an apoapsis exceeding $12700 \mathrm{~km}$ above the southern hemisphere at the beginning of the mission, lowered to about $8000 \mathrm{~km}$ after one year. The altitude change also increased the number of orbits per day, from 2 to 3 . This led to an uneven data distribution, and only measurements over the northern hemisphere are assumed to be inside the magnetospheric cavity which allow modeling of Mercury's internal magnetic field. The entire planet is covered in 59 (terrestrial) days, and all local times are sampled twice within 176 days. MESSENGER returned magnetic field measurements in the MBF (Mercury Body Fixed) and in the MSO (Mercury Sun Oriented) reference systems. These are further described in Section 2.5.

Three different data selection schemes are considered, with the goal of deriving a steady magnetic field model. First, we select all data with a satellite altitude below 1000 $\mathrm{km}$. This scheme is denoted as _alt. Second, we select data using a proxy defined by Oliveira et al. (2015) that indicates whether the measurement are taken within the magnetospheric cavity or not. This is denoted as _mag. Third, data are selected during local night time and below $1000 \mathrm{~km}$ altitude (scheme _a-n). The selection of data only during local night times is often used in satellite based geomagnetic field modeling (e.g. Lesur et al., 2015; Finlay et al., 2016), and ensures reduced external magnetospheric field strengths (Anderson et al., 2013). The altitude selection criterion guarantees that the analyzed magnetic field measurements are close enough to the surface of Mercury so that the internal magnetic field dominates the signal. The altitude limit is below the average subsolar distance of the magnetopause location (Winslow et al., 2013), which in turn should ensure the sampling of the magnetic field within a source-free region. The third data set is a subset of the second one, which is itself a subset of the first one.

\subsection{Model description}

In this study, the model of Mercury's steady field is parameterised in terms of spherical harmonics, which is a widely used technique in geomagnetic field modeling (i.e. Langel, 1987), and was applied earlier to derive models of planetary magnetic fields (i.e. Holme \& Bloxham, 1996; Anderson et al., 2008; Uno et al., 2009). This is different to recently applied approaches to model Mercury's magnetic field which sought to overcome the lack of magnetic field measurements in the southern hemisphere. For instance, Anderson et al. (2012) related magnetic equator crossings to the axisymmetric field. Oliveira et al. 
(2015) used an equivalent source dipole scheme (Langlais et al., 2004) over the northern hemisphere only. Thébault et al. (2018) favored a parameterization based on localized functions over the northern hemisphere, using the revised spherical cap harmonic analysis method (Thébault et al., 2006).

However, as it has been shown by several studies (Ness et al., 1974a; Holme \& Bloxham, 1996; Uno et al., 2009; Ridley \& Holme, 2016; Connerney et al., 2018) spherical harmonic analyses can provide robust estimates of planetary magnetic fields even from single fly-bys and un-even data distribution, provided some prior regularization. In a sourcefree region without electric currents, the steady magnetic potential is given by a spherical harmonic expansion

$$
\begin{aligned}
V= & \left.a \sum_{l=1}^{L_{\text {int }}} \sum_{m=0}^{l}\left\{\left(g_{l}^{m} \cos (m \phi)+h_{l}^{m} \sin (m \phi)\right)\left(\frac{a}{r}\right)^{l+1} P_{l}^{m}(\cos \theta)\right)\right\} \\
& +a \sum_{l=1}^{L_{\text {ext }}} \sum_{m=0}^{l}\left\{\left(q_{l}^{m} \cos (m \phi)+s_{l}^{m} \sin (m \phi)\right)\left(\frac{r}{a}\right)^{l} P_{l}^{m}(\cos \theta)\right\},
\end{aligned}
$$

where $a$ is the Mercury's mean radius (2440 km). $r, \theta, \phi$ are the MBF planetocentric coordinates of MESSENGER, $r$ the radial distance from the planetary center, $\theta$ the colatitude, and $\phi$ the longitude. The $P_{l}^{m}(\cos \theta)$ are the Schmidt normalized associated Legendre functions, where $l$ is the degree and $m$ the order. $L_{\mathrm{int}}$ and $L_{\text {ext }}$ are the truncation degrees of the spherical harmonic expansions for the internal and external field, respectively. The model parameters $\left\{g_{l}^{m}, h_{l}^{m}\right\}$ and $\left\{q_{l}^{m}, s_{l}^{m}\right\}$ are called Gauss coefficients and represent the internal and external magnetic field, respectively.

These model parameters are estimated by a least squares fit to data collected during a given time interval. For a linear inverse problem (least squares fit) the model vector $\mathrm{m}$ containing the Gauss coefficients is found at the minimum of an objective function

$$
\Theta(m)=(\mathbf{y}-\mathbf{A} m)^{\top} \mathbf{C}_{\mathbf{e}}^{-\mathbf{1}}(\mathbf{y}-\mathbf{A} \mathbf{m})+\lambda_{S}\left(\mathbf{m}^{\top} \mathbf{C}_{\mathbf{m}} \mathbf{m}\right),
$$

where $\mathbf{y}$ is the data vector, $\mathbf{A}$ a design matrix, $\mathbf{C}_{\mathbf{e}}$ the data error covariance matrix, and $\mathbf{C}_{\mathbf{m}}$ the prior model covariance matrix (Jackson, 1979; Gubbins, 1983), controlled by a Lagrange multiplier, $\lambda_{S}$. The final model represents the optimal balance between data misfit and model smoothness, which is found for the $\lambda_{S}$ at the knee of their trade-off curves.

The inverse problem is ill-posed, as a large number of observations have to be explained by a truncated set of model parameters. This leads to an ambiguity in the inversion. In general, solving ill-posed inverse problems requires regularization to reduce 
the ambiguity towards a prior constraint and to stabilize the solution of the inversion (Levenberg, 1944; Tarantola, 1987). A variety of spatial constraints could be applied to reduce the ambiguity of the inversion (see Holme and Bloxham (1996) for a discussion of spatial constraints). Here we choose to utilize a prior constraint that controls the complexity of the model field morphology at a chosen spherical surface of radius $c$. We employ

$$
\mathbf{C}_{\mathbf{m}}:\left.\oint B_{r}^{2} d S\right|_{r=c}=4 \pi \sum_{l=1}^{L_{\text {int }}} \sum_{m=0}^{l} \frac{(l+1)^{2}}{2 l+1}\left(\frac{a}{c}\right)^{(2 l+4)}\left(g_{l}^{m 2}+h_{l}^{m 2}\right)
$$

to minimize the mean square radial field at Mercury's surface, with $c=2440 \mathrm{~km}$. The diagonal elements of $\mathbf{C}_{\mathbf{m}}$ are then

$$
\operatorname{diag}\left[\frac{(l+1)^{2}}{2 l+1}\left(\frac{a}{c}\right)^{(2 l+4)}\right] .
$$

As long as $a \geq c$, the diagonal elements of $\mathbf{C}_{\mathbf{m}}$ grow with the degree of spherical harmonics. Therefore, contributions of the higher degrees of spherical harmonics are more strongly regularized during the inversion, which ensures the convergence of the norm.

Usually, in geomagnetic field modeling $c$ is set to the radius of Earth's core. This could have been adopted here by using estimates of Mercury's core radius, between $c=$ $2004 \mathrm{~km}$ and $c=2030 \mathrm{~km}$ (Rivoldini \& Van Hoolst, 2013; Hauck et al., 2013). However, there are no reason why one value or the other should be chosen. In addition, the radius of Mercury's dynamo source region is not well known. We therefore set $c=a=2440$ $\mathrm{km}$. This slightly modifies the effect of the prior constraint, as it regularizes terms of higher spherical harmonic degrees less strongly than when $c=2004 \mathrm{~km}$ or $c=2030 \mathrm{~km}$, which may influence the resulting quadrupole-to-dipole ratio (a key parameter of Mercury's magnetic field).

\subsection{Maximum degree of the model, and separation of internal and ex- ternal contributions}

The partial hemispherical coverage by the MESSENGER mission precludes large maximum degree in (1). This in turn may cause a spectral leakage, as the spectral energy of unmodelled magnetic fields, i.e. $l>L$, are indefinitely mapped onto spherical harmonic degrees $l \leq L$. Possible sources could be magnetic fields of Mercury's crust and core, but also its magnetosphere. In addition, a spectral leakage of external magnetic field energy into the internal magnetic field model coefficients may also occur and vice versa (Thébault et al., 2012). 
We perform a covariance analysis of the model inversion (see appendix A). Results (Fig. A.1) reveal significant correlations among coefficients across spherical harmonic degrees. The clearest ones are for $\left(g_{1}^{0}, g_{2}^{0}\right),\left(g_{1}^{1}, g_{2}^{1}\right),\left(h_{1}^{1}, h_{2}^{1}\right)$, and $\left(g_{1}^{0}, g_{3}^{0}\right)$. Odd terms $(l$ $=1$ and $l=3)$ are correlated, and anti correlated to even terms $(l=2)$. Because of that, these coefficients can not be robustly and independently estimated. Their dependence can be directly related to the geometrical similarity of their spatial sensitivity, as well as to the uneven distribution of the MESSENGER data.

The covariance analysis of the model also shows that there is no significant spectral leakage between external and internal field coefficients (Fig. A.1). Those seem to be mostly independent of each other. We conclude that there is a good separation between external and internal magnetic field sources.

We set the maximum spherical harmonic degree of the internal field $L_{\text {int }}$ to 3 in (1). This choice is made because we seek to model only the large scale and internal magnetic field. A choice with $L_{\text {int }}=1$ could also have been considered. However, then the model would not have allowed to derive the quadrupole-to-dipole ratio of Mercury's magnetic field, a key parameter.

Concerning the external field, derivation and interpretation of models with $L_{\text {ext }}>$ 1 are uncertain, because the convergence of (4) is not guaranteed, as the norm (4) is not bounded anymore $(c>a)$, and therefore with no control on small scale external field contributions. The external field estimation of even vs. odd spherical harmonic degrees is also subject to the uneven spatial distribution of MESSENGER data. To this end, we set the maximum spherical harmonic degree of the external field $L_{\text {ext }}$ to 1 , without using any prior constraint.

\subsection{Inversion results}

We present in Tab. 1 the number of selected measurements for each selection scheme, and the misfit of the associated models. Table 2 lists the coefficients of the different steady field models in the MBF coordinate system and also reports those of Anderson et al. (2012) and Thébault et al. (2018).

The best fit to the measurements is found for model _a-n. This is mainly because this selection scheme rejects day side measurements, limiting the effect of the external 
field which is widely reduced during night local times. The other data selection schemes, i.e. _alt and _mag, do not suspend local day time data from the model derivation, explaining their larger associated misfits. When compared to previously published models, the best agreement is found between the global internal field description of Thébault et al. (2018) and the model based on data selection scheme _a-n. The external field coefficients; however differ and indicate that the night side external field is on average less intense. Differences between models_alt, _mag and that of Thébault et al. (2018) can be explained by similar reasons: they are likely due to magnetic fields generated by electrical currents flowing at day times in the plasma environment of Mercury, that are not excluded by the data selection. We finally note that models_alt and _mag have similar internal field coefficients as the model by Anderson et al. (2012) which is based on the first 9 months of measurements, and for which data include all local times.

Our preferred Model is model _a-n, as it provides the best estimate of Mercury's large scale steady internal field. By using estimates of the covariance matrix we can also compute a formal error associated with the Gauss coefficients (Bloxham et al., 1989). This error or uncertainty is found to be $\sim 6 \%$ for each coefficient.

\subsection{Maps of the residual fields}

Before the temporal variability of the residual magnetic field is studied in detail, we describe the spatial characteristics of the residual field. Residuals, $\delta \mathbf{B}$, between MESSENGER measurements and the steady field model, $\mathbf{B}_{\mathbf{M}}$, are computed using model _a$\mathrm{n}$,

$$
\delta \mathbf{B}=\mathbf{B}-\mathbf{B}_{\mathbf{M}} .
$$

These residuals are computed in both the MBF and MSO reference frames. The conversion from the MBF residual components $\left(\delta B_{r}, \delta B_{\theta}, \delta B_{\phi}\right)$ to the MSO ones $\left(\delta B_{x}, \delta B_{y}\right.$, $\left.\delta B_{z}\right)$, with $x$ pointing towards the Sun, follows:

$$
\begin{aligned}
\delta B_{x} & =\delta B_{r} \sin \theta_{S} \cos \phi_{S}+\delta B_{\theta} \cos \theta_{S} \cos \phi_{S}-\delta B_{\phi} \sin \phi_{S}, \\
\delta B_{y} & =\delta B_{r} \sin \theta_{S} \sin \phi_{S}+\delta B_{\theta} \cos \theta_{S} \sin \phi_{S}+\delta B_{\phi} \cos \phi_{S}, \\
\delta B_{z} & =\delta B_{r} \cos \theta_{S}-\delta B_{\theta} \sin \theta_{S} .
\end{aligned}
$$

$\phi_{S}$ and $\theta_{S}$ are solar longitude and latitude. 
Residuals in the MBF system for ascending (pole ward) orbital legs during one Hermean year ( 88 terrestrial days between two consecutive perihelions of Mercury) are shown in Fig. 1. Those in the MSO system for the same time-span are also displayed in the same figure (bottom panel). The maps in the MBF coordinate system do not show a simple residual field morphology. The considered time period corresponds to 1.5 full tour of MESSENGER around Mercury. The western hemisphere (negative longitude) is covered twice. The overlapping field residuals are actually very different, with negative and positive features sensed over the same location but at different epochs. This strongly suggests that these residuals cannot be associated with steady internal sources. This conclusion was also reached by Johnson et al. (2012); Korth et al. (2015). When these residuals are plotted in the MSO reference frame, they show a different organization. The $\delta B_{x}$ residuals show moderate to large positive amplitudes and arrange in a circular pattern centered in the North pole with an approximate latitudinal range from 60 to 87 degrees. The $\delta B_{y}$ residuals (same Figure, middle panel) show a noticeable and regular pattern of positive and negative amplitudes centered around the North pole. This may suggest a substantial unmodelled small scale contribution of axial symmetry, i.e. possibly of degree 4 and order 2. In the midday to dusk section (longitudes from 0 to $-90^{\circ}$ ) and in the midnight to dawn section (longitudes from 180 to $90^{\circ}$ ) significant residuals exist in a latitudinal band close to the equator. The map of $\delta B_{z}$ (right panel) shows a region with large positive residuals at the day-side. This region starts slightly after sunrise and extends somewhat into the night-section. Near the North pole two small areas with opposite polarity are found, and may be related to processes in the polar cusp, and possibly linked to Birkeland currents, which are signatures of a magnetospheric circulation (Slavin et al., 1997; Anderson et al., 2014). These large scale residual patterns are also found in most other epochs of the mission's lifetime, with positions fixed with respect to the Sun.

\section{Model of the time-varying residual fields}

We now turn to the modeling of these unmodelled magneticfield by subtracting the steady field MBF_a-n model from MESSENGER's magnetic field measurements below $1000 \mathrm{~km}$ altitude by using (6). Here, we do not discard measurements on the day side, nor do we use the criterion defined by Oliveira et al. (2015). The set of residual data is also fitted by spherical harmonic expansions, i.e. (1). As discussed in section 2.3 the deriva- 
tion of external field models with of $L_{\text {ext }}>1$ is omitted. For the internal field, we set $L_{\text {int }}$ to 3 . This choice is consistent with the approach taken for the steady fields.

Instead of inverting the entire data set at once, it is sorted in sub-samples of a given length as defined below. This approach allows to derive time series of internal and external Gauss coefficients. The length or duration of each sub samples is defined using the following requirements: (1-a) have an optimal temporal resolution; (1-b) have a good spatial coverage. These are needed to construct (2-a) robust estimates of spatially large scale external and internal field contributions, leading to (2-b) characterize significant temporal magnetic field variations. With these objectives, we tested different temporal sampling and grouping of the data to invert them in terms of time series of Gauss coefficients. Different settings of the data sampling are examined, some for which the data are sorted into overlapping or non-overlapping segments of 2 to 20 days length (temporal sub-sampling). We also tested an orbital sub-sampling, where the data set is sorted to keep a constant number of consecutive orbits. Generally, it is found that data sets covering longer time intervals show weaker temporal variability of the derived individual Gauss coefficients, likely because variations cancel out over longer time span. Orbital sub-sampling leads to an uneven temporal resolution, with Gauss coefficients derived every 2.5 to 5 days, depending on the number of orbits per day or on possible data gaps, while providing a more even spatial sampling than the temporal sub-sampling. For the latter reason we prefer orbital sub-sampling of the residual data set, and find that non-overlapping sets of 8 orbits provide reasonable inversion results. Figure 2 shows the residual field components after the further subtraction of time varying fields in MBF and MSO coordinate systems for the same time period as in Figure 1. Amplitudes of the remaining field are significantly reduced. The rms misfit of all 8-orbits samples ranges between $4 \mathrm{nT}$ and $30 \mathrm{nT}$, and its average is approximately $16 \mathrm{nT}$. Remaining structures are caused by unmodelled fields with, perhaps, different sources.

In the following we analyze coefficients of the first spherical harmonic degree only. Coefficient time series of the varying field consist of $\delta g_{1}^{0}(t), \delta g_{1}^{1}(t), \delta h_{1}^{1}(t)$ for the internal field and $\delta q_{1}^{0}(t), \delta q_{1}^{1}(t), \delta s_{1}^{1}(t)$ for the external field. Their formal error ranges between 9 and $12 \%$ for the internal coefficients, while those of the external field tend to be somewhat smaller. 
To allow for a time series analysis (Section 4) we interpolate series of Gauss coefficients with a spline function (de Boor, 1978), and compute a regular temporal division of one day. We note that the resulting temporal resolution of the time series remains close to 5 days.

\subsection{Inversion results for the time-varying coefficients}

Fig. 3 shows time series of the 6 Gauss coefficients of Mercury's time varying internal and external magnetic fields computed from residuals in the MBF coordinate system. The series of axial components, $\delta g_{1}^{0}$ and $\delta q_{1}^{0}$ (shown in the top panel of Fig. 3) seem to oscillate with a common period and show a fixed phase relation. Both coefficients show also a long term variability. It is clearly seen as an amplitude variation with smaller amplitudes between 2013.0 and 2013.5, and larger amplitudes at the beginning and end of the mission. There is an apparent absolute shift between the axial coefficients, the internal one being most of the time about $20 \mathrm{nT}$ larger than the external one. The internal equatorial terms (bottom panel, Figure 3 ), $\delta g_{1}^{1}$ and $\delta h_{1}^{1}$, show similar amplitudes, which vary over time and with slightly larger amplitudes around 2013.0 - 2013.5. Their amplitudes are smaller than those of the external equatorial terms $\delta q_{1}^{1}$ and $\delta s_{1}^{1}$. Furthermore, the variation of equatorial external and internal terms show more complex phase relations.

The cause for the long-term variation of $\delta g_{1}^{0}$ and $\delta q_{1}^{0}$ could be related to the varying geometry of MESSENGER's orbit over the mission period. Around 2013.2 the periapsis of MESSENGER reached its northernmost latitude. At this epoch the data distribution was more or less symmetric on the ascending and descending legs of the orbits, i.e., with a similar number of measurements on the day and night sides. Before and after this epoch, individual orbital legs were dominated by the descending and ascending part, respectively. In other words, there were more data on one or the other side of the planet, either day or night depending on the epoch. We investigated the effect of the symmetric or not-symmetric data distribution on the resulting coefficients and performed a covariance analysis (see appendix A). Although the covariance matrix varies with time, there is no clear relationship with the varying periapsis latitude. 


\subsection{Magnetic dipole moments}

In order to compare the internal and external magnetic field contributions, we compute their mean surface value, similarly to the approach of Grosser et al. (2004). For the internal field, it is

$$
M_{\mathrm{int}}=\sqrt{(l+1) \sum_{l=1}^{L_{\mathrm{int}, \max }} \sum_{m=0}^{m=l}\left[\left(g_{l}^{m}\right)^{2}+\left(h_{l}^{m}\right)^{2}\right] .}
$$

For the dipole, $L_{\mathrm{int}, \max }=1$, this quantity becomes the dipole moment and is expressed as

$$
M_{\mathrm{int}}(t)=\sqrt{2 *\left(\left(g_{1}^{0}+\delta g_{1}^{0}\right)^{2}+\left(g_{1}^{1}+\delta g_{1}^{1}\right)^{2}+\left(h_{1}^{1}+\delta h_{1}^{1}\right)^{2}\right)} .
$$

The dipole moment of the static or time-averaged internal field is

$$
\bar{M}_{\mathrm{int}}=\sqrt{2 *\left(\left(g_{1}^{0}\right)^{2}+\left(g_{1}^{1}\right)^{2}+\left(h_{1}^{1}\right)^{2}\right)} .
$$

The time-varying internal dipole moment is finally defined by

$$
\delta M_{\mathrm{int}}(t)=M_{\mathrm{int}}(t)-\bar{M}_{\mathrm{int}} .
$$

For the dipole moment of the external field the expression is

$$
M_{\mathrm{ext}}(t)=\sqrt{l \sum_{l=1}^{1} \sum_{m=0}^{m=l}\left[\left(q_{l}^{m}\right)^{2}+\left(s_{l}^{m}\right)^{2}\right]},
$$

Our definition of dipole moments is in accordance with the field energy (Mauersberger, 1956; Lowes, 1966) and differs from the definition given by Grosser et al. (2004), who used the factor $(l+1)$ in (12). While these dipole moments are dominated by axial terms, they also take into account the equatorial ones, and as such, are more complete proxies of the large scale internal and external field temporal variations at Mercury.

Figure 5 shows series of the external and internal magnetic dipole moments at the planet surface. The variation of both is similar and apparently in phase. The amplitude of $M_{\text {ext }}(t)$ ranges from 20 to $120 \mathrm{nT}$, and the amplitude of $\delta M_{\mathrm{int}}(t)$ is between $\pm 70 \mathrm{nT}$. These values have to be compared to $\bar{M}_{\text {int }}$, which we estimated at $305 \mathrm{nT}$ for the dipole.

\section{Results}

\subsection{Comparison with Mercury's orbit evolution}

We now compare the temporal variability of the external magnetic dipole moment with the position of Mercury around the Sun, i.e. its heliocentric distance, in Fig. 6. The 
external field varies with the heliocentric distance, but does not show a constant phase relation with the heliocentric distance. Prior to 2013.0 variation of the heliocentric distance runs ahead variations of $M_{\text {ext }}(t)$. Maxima of $M_{\text {ext }}(t)$ occur slightly before Mercury's perihelion. After 2014.0, maxima occur shortly later than the perihelion. We interpret this phase change to be related to the evolution of MESSENGER's orbital geometry.

The plots in the bottom part of Fig. 6 show MESSENGER's orbits for selected epochs in the MSO reference frame. For the epoch 2011.49 a minimal external field is observed when MESSENGER had its lowest measurement point on the night side. We find a maximum of the external field at 2011.64, when MESSENGER's periapsis is at day. However, the latitude of the MESSENGER's periapsis is not constant with Mercury's rotation period (59 days). The same latitude of the periapsis and the same local time is reached approximately every 54 days. It is suspected that such a latitudinal difference could translate into a temporal shift due to a hemispherical magnetic asymmetry. For instance, magnetic field patterns that may only exist in a confined latitudinal range. A periapsis of MESSENGER over this region would occur on different local times, for which the strength of these magnetic fields may be different. When MESSENGER had its periapsis close to the north pole, the lowermost measurements were close to dawn and dusk. This may explain why the minimum and maximum external fields are less intense.

\subsection{Results of the spectral analysis}

We now turn to the spectral analysis of Mercury's external and internal field variability (details of the method are provided in appendix B). Figure 7 shows power spectra of the dipole Gauss coefficients representing the large scale of Mercury's internal and external time-varying magnetic fields. Several spectral peaks can be identified. We mark 6 different significant periods in the individual spectra with colored bars. These periods are related to Mercury's orbital period of 88 days (annual period) and its first two subharmonics at 44 and 29 days, the rotation period of 59 days, and Mercury's length of solar day of 176 days, respectively. The synodic rotation of the Sun as seen from Mercury, at 36 days, also shows a peak.

Peaks show different spectral strengths depending on the coefficients. For instance the synodic period (orange bar) is significant in the equatorial coefficients of the inter- 
nal and external field but not in the axial ones. Signals related to the orbital period (44 and 88 days) are significant in the axial field components and faintly appear in the equatorial terms. These spectral peaks show a prominent doublet structure. The 176-days period causes a noticeable peak in all spectra, but significant peaks are found only for the equatorial terms of external and internal fields. These peaks tend be slightly broader, which may reflect a slightly poorer sensitivity of the spectral estimation towards longterm cycles that are only a few times accommodated in the time series.

Spectra of the varying internal and external dipole moments $\delta M_{\text {int }}(t)$ and $M_{\text {ext }}(t)$ are shown in Figure 8. Distinct spectral peaks can be identified and we mark the same periods in the individual spectra with colored bars as in Figure 7. Significant peaks are related to Mercury's orbital period and its harmonics at 44 and 29 days (dark magenta bars), whereas other periods show no significant peaks. These peaks show, again, a doublet structure. We interpret this as being caused by the slight phase change that occurred around the middle epoch of the mission, when MESSENGER reached its most northern periapsis around 2013.2. This is further confirmed when performing spectral analyzes separately before and after 2013.2, then peaks associated with the orbital period and its harmonics appear as single peaks.

\subsection{Results of the coherence analysis}

Significant periods which are identified in both dipole moments series may provide an appropriate measure to diagnose mutual coherent behavior using methods of coherence analysis (see appendix B for details). Figure 9 shows coherence and phase spectra of the two time series $\delta M_{\text {int }}(t)$ and $M_{\text {ext }}(t)$. The coherence spectrum is very detailed and shows numerous significant spectral peaks, which mostly relate to the spectral bands observed in the individual series (Figure 8). The annual variation (88 days) is significant in individual spectra of the magnetic dipole moments, and so is its MTM-coherence. The semi-annual variation (44 days) is found in the internal and external dipole moment variations, but it causes no notable peak in the coherence, unlike the 29-day period.

There are also coherent peaks which do not exist in the spectra of the individual series. Most prominent are 5 such peaks in a period range between 88 and 176 days. Possibly, these peaks are caused by superposition or combination of different periods. For instance, a superposition of the annual and the semi-annual period could lead to a vir- 
tual period of 132-days, which is observed. It is found that these combinations involve all periods identified in Figure 8, even though they are not significant in the individual spectra of the dipole moments.

The phase spectrum is shown in Figure 9 (bottom panel). Non-zero phase angles indicate a leading or a trailing of $M_{\text {ext }}(t)$ with respect to $\delta M_{\text {int }}(t)$, if the angle is positive or negative. The phase angle refers also to a lag-time, which depends on the given coherent period. The light-colored region in the phase spectrum displays the area of phase uncertainty of the 0 degree phase angle. This area is determined by the sub-sampling of the residual data into 8-orbits sample and by the applied spline interpolation in section 3. A phase angle within this area is not resolved by our analysis. None of the significant coherent periods show angles which are outside light-colored region. This means that lag-times can not be clearly resolved by the analysis. We therefore interpret the phase angles of all coherent periods to be indistinguishable from zero, and that lag-times are shorter than 5 days for these coherent periods. Variations in $\delta M_{\text {int }}(t)$ and $M_{\text {ext }}(t)$ can be assumed to be coincident.

Finally, Figure 10 shows the wavelet coherence between $\delta M_{\text {int }}(t)$ and $M_{\text {ext }}(t)$ series. Significant coherence is mainly observed in a period range from 70 to 140 days, with a center at the 88-days period. The widening of this band is likely caused by the superposition of the 88-days period with other periods, as discussed for the results. Further coherence patches are also found for shorter periods, but they appear to be discontinuous in time.

Most noticeable is the gap between patches of significant coherence in the middle of observation period around day 720, i.e. around 2013.2. The extent of this gap is roughly 100 days, but may vary depending on the significance level applied for the wavelet coherence.

From the coherence analyses we conclude that MTM-coherence and wavelet-coherence reveal coherent temporal variability of the internal and external dipole moments. Significant coherence exists in an approximate period range from 60 to 150 days. This confirms the coherent temporal behavior related to the 88-days period, i.e., the period of Mercury's motion around the Sun. Other periods show no clear coherence. Coherent variations show no phase angles and appear to be simultaneous. 


\section{Discussion and implications}

Results of this study may hold implications for our understanding of different magnetic field generation processes that are sampled by MESSENGER's magnetometer data. In the following, we discuss results of the steady magnetic field modeling and the residual field analysis.

\subsection{The steady magnetic field}

Perhaps, one important result that can be derived from our steady field model is the quadrupole-to-dipole ratio. We find $g_{2}^{0} / g_{1}^{0}=0.27$, which is in agreement with the value reported by Thébault et al. (2018), but use a different modeling method. The reason why our value of the $g_{2}^{0} / g_{1}^{0}$ ratio and that of Thébault et al. (2018) largely differ from the value of Anderson et al. (2012) may be explained by several reasons. One is related to the data selection. Anderson et al. (2012) considers data from all local times in the model derivation, whereas this study uses only local night time data below $1000 \mathrm{~km}$ altitude to derive the internal magnetic field model. The selection of night time data reduces contributions from external fields, which have a significant impact on internal field coefficients. This can clearly be seen in Table 2, where we compare models derived from all local times and night time data. However, we note that Thébault et al. (2018) did not specifically reject day side measurements, so this is not the only explanation. The far-field modeling technique, as applied by Anderson et al. (2012) to derive their magnetic field model might be prone to current systems in Mercury's magnetosphere and their related magnetic fields. This approach may also favor a high quadrupole-to-dipole ratio as it emphasizes equatorial data and down-weight data over polar regions (Thébault et al., 2018). We note that Anderson et al. (2008) obtained a lower quadrupole-to-dipole ratio which is comparable to ours, when the magnetospheric magnetic field is accounted by using an empirical model of the magnetopause and tail currents similar to that of Tsyganenko and Sitnov (2005). Therefore the true quadrupole-to-dipole ratio may still be a matter of debate, as the non-uniqueness imposed by MESSENGER's data distribution critically hampers the determination of the spherical harmonics even degree terms, and therefore affects $g_{2}^{0} / g_{1}^{0}$ ratio.

We additionally derive the ratio of dipole to non-dipole axial terms by

$$
D=\frac{\left|g_{1}^{0}\right|}{\sqrt{\left(g_{2}^{0}\right)^{2}+\left(g_{3}^{0}\right)^{2}}} .
$$


The ratio provides a simplified measure of the magnetic field dipolarity (Christensen et al., 2010). It is $D=3.2$ for the model MBF_a-n and it deviates from the value derived from the coefficients given by Anderson et al. (2012), that is $D=2.5$. Earth's value derived from the IGRF (International geomagnetic reference field Thébault et al., 2015) at epoch 2015 is $D=6.2$. This value is computed at the same relative distance from the liquid core, as for Mercury. The values for Mercury largely differ from Earth's value, indicating that Mercury's magnetic field is less dipolar than Earth's magnetic field. The so-called dipole offset is, therefore, a characteristic feature of Mercury's low magnetic field dipolarity. Values of the dipolarity based on model by Anderson et al. (2012) and those derived from this study differ.

\subsection{Time-varying magnetic fields}

The relevance of our results of the time-varying modeling relies on the correctness of our assumption that measurements are acquired in a magnetic source-free region. This is likely to be the case, as the altitude range below $1000 \mathrm{~km}$ is negligibly populated by Ions. Only Birkeland currents are expected to exist at low altitudes in a confined cone of $15^{\circ}-30^{\circ}$ in colatitude around the North pole (Anderson et al., 2014).

In our spectral analyses of the external and internal dipole moment variation that are mostly determined by the variation of $\delta g_{1}^{0}$ and $\delta q_{1}^{0}$ (see (11)), we identify 3 spectral peaks, which are related to the 88-days orbital period of Mercury, i.e., the annual periodicity and its sub-harmonics. The identified 29-days period is indeed the second subharmonic of Mercury's orbital period, as well as it could be the first sub-harmonic of the Mercury's rotation period at 59 days. However, this period is not found in the spectral analyzes and therefore should not show sub-harmonics. These lead to the conclusion that the temporal variability of the internal and external residual fields is tightly linked to Mercury's orbital motion around the Sun.

Similar conclusions have been reached by previous studies (Suess \& Goldstein, 1979; Winslow et al., 2013; Johnson et al., 2016; Korth et al., 2017), where external field variations are found to be related to the magnetopause stand-off distance. It varies with the planet's heliocentric distance and the changing solar wind pressure during the planet's orbit generates a varying external magnetic field around Mercury. The doublet peaks of annual and semi-annual variations in the spectra of the axial dipole coefficients and 
the dipole moments (Figs. 7 and 8) disappear when the analysis is ran separately for periods prior or after 2013.2. Therefore, the split-up is possibly related to the phase shift seen in Figure 6 which is a consequence of the changed orbital geometry of MESSENGER. External field variations seem to be fixed with respect to the Sun and do not corotate with Mercury, as we find no significant signals related to the 59-days period (sidereal rotation period).

Another feature is the low variability of the axial terms and dipole moments around 2013.2, see Figure 3. The wavelet coherence of $\delta M_{\text {int }}(t)$ and $M_{\text {ext }}(t)$ in Figure 10 displays a distinct gap centered around 2013.2, which can be related to MESSENGER's most northern periapsis.

\subsection{Electrical conductivity of the mantle}

Overall, the synchronous behavior of external and internal field variations at coherent periods suggests a possible interpretation that involves the induction of internal magnetic fields due to external magnetic field variations. Such effect has been studied previously (Grosser et al., 2004; Heyner et al., 2016; Johnson et al., 2016).

If we assume the variation of the internal magnetic dipole moment to be dominantly caused by an induction process in the core driven by external field variations, then implications for the electrical mantle conductivity can be derived. Following Suess and Goldstein (1979), the characteristic time for the external field to diffuse through Mercury's mantle to the core is given by:

$$
\tau_{D} \approx \mu_{0} \sigma_{M} \mathrm{~L}^{2},
$$

where $\mu_{0}$ is the permeability of free space, $\sigma_{M}$ the electrical mantle conductivity and $\mathrm{L}$ $=440 \mathrm{~km}$ the mantle thickness, respectively. This characteristic time corresponds to the delay that would be observed between the inducing external field and its induced internal counterpart.

By using (14), we can derive an upper limit of the electrical mantle conductivity. All reported temporal variations are highly correlated and associated with a time-lag which, if it exists, is below our sensitivity of 5 days. This corresponds to an upper limit of $\sim$ $1 \mathrm{~S} / \mathrm{m}$. Shorter time-lags, yet unresolved by our approach, would correspond to smaller values of the electrical mantle conductivity. The electrical conductivity of Earth-like materials like olivine and magnetite ranges from $10^{-4} \mathrm{~S} / \mathrm{m}$ to $10^{3} \mathrm{~S} / \mathrm{m}$ at $300 \mathrm{~K}$ (Parkin- 
son \& Hutton, 1989). Our result agrees with synthetic electrical conductivity profiles of Mercury's mantle and crust which range from $10^{-4} \mathrm{~S} / \mathrm{m}$ to $1 \mathrm{~S} / \mathrm{m}$ for different scenarios of Mercury's formation by Verhoeven et al. (2009).

\subsection{Mercury's core size}

We now attempt to derive the Mercury's core size and adopt a formalism that was derived by Grosser et al. (2004), which expands the study of Rikitake (1966). Their study showed, that for an exciting external magnetic field variation, $B_{\text {exc }}$, with periods of the order of 1 second and longer, the ratio of internally induced $\left(B_{\text {ind }}\right)$ to the exciting external magnetic fields can be approximated by:

$$
\frac{B_{\text {ind }}}{B_{\text {exc }}}=\frac{n}{n+1}\left(\frac{r_{c}}{a}\right)^{2 n+1} .
$$

Our results show that external and internal magnetic fields are correlated with a period close to 88 days (Figs. 7, 8, 9 and 10). Such a relationship can also be represented by Gauss coefficients (Olsen, 1999; Tarits \& Grammatica, 2000), particularly when the external and internal fields can be largely described by a single coefficient, like axial dipole terms. But this fails for Mercury, where equatorial terms cannot be ignored. It is, therefore, necessary to use magnetic dipole moments ((8) to (12)). However, one has also to take into account the internal magnetic field moment related to dynamo processes (10), and to consider instead the time-varying one (11). This poses a further complication, as the external dipole moment $M_{\text {ext }}(t)$ is always positive, while the internal $\delta M_{\text {int }}(t)$ has both positive and negative values, with a close-to-zero average. For these reasons we consider $B_{\text {exc }}$ and $B_{\text {ind }}$ in (15) to be equivalent with $M_{\text {ext }}(t)$ and $\delta M_{\text {int }}(t)$ only for epochs when those two terms are positive, i.e., for 259 epochs (out of 507). The arithmetic mean values are found to be 19.8 and $65.7 \mathrm{nT}$ for internal and external parts, respectively.

Rearranging (15), and introducing the mean time-varying internal and external dipole moments, i.e., $n=1$, we find Mercury's core radius to be

$$
r_{c}=a\left(\frac{n+1}{n} \frac{B_{\mathrm{ind}}}{B_{\mathrm{ext}}}\right)^{1 /(2 n+1)}=a\left(2 \frac{\left|\delta M_{\text {int }}(t)\right|}{\left|M_{\text {ext }}(t)\right|}\right)^{1 / 3}=2060 \mathrm{~km} .
$$

This value has to be seen as that of the electrically conductive core of Mercury, i.e., not necessarily that of the dynamo nor of its liquid part. In order to derive an error bar, we use the uncertainty estimates of the inversion, which yields an average formal error of $10 \%$ for each coefficients. This is slightly more than for the static, mean field model, as 
the static model is based on a much larger data set. Assuming the magnetic field coefficients to be Gauss-distributed random variables, the uncertainty range of dipole moments is $\sim 17.3 \%$. The uncertainty of the core radius estimates, which is based on 259 samples, becomes $0.173 / \sqrt{258}=1.1 \%$. This is approximately $\pm 22 \mathrm{~km}$.

Our result $(2060 \pm 22 \mathrm{~km})$ is consistent with the result of Johnson et al. (2016) who gave a conductive core radius range of [1900-2060 km] or [2020-2200 km], depending on the external field magnetic field model. Although it is also independent from geodetic observations, it is consistent with results of Rivoldini and Van Hoolst (2013) and Hauck et al. (2013), who found values of $2004 \pm 39 \mathrm{~km}$ and $2020 \pm 30 \mathrm{~km}$ for the liquid core radius, respectively.

\section{Conclusion}

In this study, we derive robust models of the steady external and internal magnetic fields of Mercury, based on a spherical harmonic analysis. External and internal magnetic fields can clearly be separated. Our preferred model agrees with previous descriptions of a strong axisymmetric internal field. The model also show a quadrupole-to-dipole ratio of approximately 0.27 , that is very similar to the value reported by Thébault et al. (2018), though our modeling approach fundamentally differs.

To study the time-varying magnetic fields of Mercury, we derive magnetic field residuals from magnetic field measurements and our preferred steady magnetic field model up to degree and order 3. A time-varying model is derived from magnetic field residuals, which are sorted into temporal bins so that each bin contains 8 consecutive orbits (provided that there are no significant gaps between orbits). For each subset, a spherical harmonic degree 3 internal and spherical harmonic degree 1 external magnetic field model is computed. The misfit of each subset significantly improves, decreasing from about $26 \mathrm{nT}$ (after the removal of the steady magnetic field) to an average of 15 and as low as $4 \mathrm{nT}$, depending on the epoch. A covariance analysis indicate a robust separation of the time-varying external and internal magnetic field coefficients. These individual models form the time series of the time-varying internal and external field coefficients.

We analyze their temporal variability. We adopt the multi-taper method to estimate the spectra of the temporal variability of the internal and external magnetic field, and to detect mutual coherent signatures in the series. A wavelet method is also applied 
to identify coherent signatures and their transient behavior. Mercury's external and internal fields show significant variations related to its orbital motion around the Sun and solar rotation. These variations are coherent and synchronous and indicate the external field variation induces internal magnetic field within Mercury. The absence of phase lags between the exciting magnetic field variations and their induced responses allows us to place an upper limit on the electrical mantle conductivity of about $1 \mathrm{~S} / \mathrm{m}$. Based on the amplitude ratio of exciting external field variation and the internally induced magnetic field, we estimate Mercury's core size to be $r_{c}=2060 \pm 22 \mathrm{~km}$. This value is in very good agreement with core size estimates from geodetic observations of Mercury's gravity field.

Some features of this study remain not fully understood, and relate to the low variability of dipole moments during a time interval centered around 2013.2. Likely, this longterm variation of the magnetic dipole moments is related to the absence of coherent variations during this time interval, as seen in Figure 10. The change of MESSENGER's orbit geometry could explain the long-term variability of the dipole moments, but not their different amplitudes. Therefore, to what extent this can be explained by the change of MESSENGER's orbit geometry needs to be understood, and if there is a common cause for these features. The un-even data distribution over the planet's hemispheres restricts conclusions from our analysis, but this will be overcome by the BepiColombo mission, which will sample Mercury's magnetic field evenly.

\section{Acknowledgments}

The MESSENGER mission was supported by the NASA Discovery Program under contracts NAS5-97271 to The Johns Hopkins University Applied Physics Laboratory and NASW-00002 to the Carnegie Institution of Washington. All MESSENGER data used here are publicly available on NASA's Planetary Data System (PDS) at http://ppi.pds .nasa.gov. This research is supported by the French Agence Nationale de la Rercherche, project MARMITE, contract 654 ANR-13-BS05-0012, as well as by Centre National des Etudes Spatiales in the context of the BepiColombo MAG experiment. The authors would like to thank Daniel Heyner for stimulating discussions, A. Rösch and H. Schmidtbauer for their help on Waveletcomp. All graphics were produced using opensource software, GMT, gnuplot and R-packages.

The authors declare that they have no competing interests. 


\section{References}

Aharonson, O., Zuber, M. T., \& Solomon, S. C. (2004). Crustal remanence in an internally magnetized non-uniform shell: a possible source for Mercury's magnetic field? $\quad$ Earth Planet. Sci. Lett., $218 . \quad$ doi: 10.1016/ $\mathrm{S} 0012-821 \mathrm{X}(03) 00682-4$

Anderson, B. J., Acuña, M. H., Korth, H., Purucker, M. E., Johnson, C. L., Slavin, J. A., ... McNutt, R. L. (2008). The Structure of Mercury's Magnetic Field from MESSENGER's First Flyby. Science, 321, 82-85. doi: $10.1126 /$ science. 1159081

Anderson, B. J., Johnson, C. L., \& Korth, H. ～(2013). ～A magnetic disturbance index for Mercury's magnetic field derived from MESSENGER Magnetometer data. Geochemistry, Geophysics, Geosystems, 14(9), 3875-3886. doi: 10.1002/ggge.20242

Anderson, B. J., Johnson, C. L., Korth, H., Slavin, J. A., Winslow, R. M., Phillips, R. J., ... Solomon, S. C. (2014). Steady-state field-aligned currents at Mercury. Geophys. Res. Lett., 41, 7444-7452. doi: 10.1002/2014GL061677

Anderson, B. J., Johnson, C. L., Korth, H., Winslow, R. M., Borovsky, J. E., Purucker, M. E., .. McNutt Jr., R. L. (2012). Low-degree structure in Mercury's planetary magnetic field. J. Geophys. Res., 117. doi: 10.1029/2012JE004159

Bloxham, J., Gubbins, D., \& Jackson, A. (1989). Geomagnetic secular variation. Philos. Trans. R. Soc. London A, 329, 415-502.

Chapman, S., \& Ferraro, V. C. A. (1940). The theory of the first phase of a geomagnetic storm. Ter. Mag. and Atm. Elec., 45. doi: 10.1029/TE045i003p00245

Chapman, S., \& Ferraro, V. C. A. (1941). The Geomagnetic Ring-Current: I Its Radial Stability. Ter. Mag. and Atm. Elec., 46. doi: 10.1029/TE046i001p00001

Christensen, U. R. (2006). A deep dynamo generating Mercury's magnetic field. Nature, 444. doi: 10.1038/nature05342

Christensen, U. R., Aubert, J., \& Hulot, G. (2010). Conditions for Earth-like geodynamo models. Earth Planet. Sci. Lett., 296(3-4), 487-496. doi: \{10.1016/j.epsl $.2010 .06 .009\}$

Connerney, J. E. P., Kotsiaros, S., Oliversen, R. J., Espley, J. R., Joergensen, J. L., Joergensen, P. S., ... Levin, S. M. (2018). A New Model of Jupiter's Magnetic Field From JUNO's First Nine Orbits. Geophys. Res. Lett., 45. doi: 
10.1002/2018GL077312

de Boor, C. (1978). A practical guide to splines. Springer-Verlag, New York.

Finlay, C. C., Lesur, V., Thébault, E., Vervelidou, F., Morschhauser, A., \&

Shore, R. (2016). Challenges Handling Magnetospheric and Ionospheric Signals in Internal Geomagnetic Field Modelling. $\quad$ Space Sci. Rev.. $\quad$ doi: $10.1007 / \mathrm{s} 11214-016-0285-9$

Ghil, M., Allen, M. R., Dettinger, M. D., Ide, K., Kondrashov, D., Mann, M. E., ... Yiou, P. (2002). Advanced spectral methods for climatic time series. Rev. Geophys., 40, 1-41.

Glassmeier, K.-H. (2000). Currents in Mercury's Magnetosphere. Geophys. Mono. Series, 118. doi: 10.1029/GM118p0371

Glassmeier, K.-H., Auster, H.-U., \& Motschmann, U. (2007). A feedback dynamo generating Mercury's magnetic field. Geophys. Res. Lett., 34. doi: 10.1029/ 2007GL031662

Gómez-Pérez, N., Heimpel, M., \& Wicht, J. (2010). Effects of a radially varying electrical conductivity on 3D numerical dynamos. Phys. Earth Planet. Int., 181. doi: $10.1016 /$ j.pepi.2010.03.006

Gómez-Pérez, N., \& Solomon, S. C. ～(2010). Mercury's weak magnetic field: A result of magnetospheric feedback? Geophys. Res. Lett., 3\%. doi: 10.1029/2010GL044533

Grinsted, A., Moore, J. C., \& Jevrejeva, S. (2004). Application of the cross wavelet transform and wavelet coherence to geophysical time series. Nonlin. Proc. Geophy, 11, 561-566.

Grosser, J., Glassmeier, K.-H., \& Stadelmann, A. (2004). Induced magnetic field effects at planet Mercury. Planet. Spa. Sci., 52. doi: 10.1016/j.pss.2004.08.005

Gubbins, D. (1983). Geomagnetic field analysis - i. stochastic inversion. Geophys. J. R. Astr. Soc., 73, 641-652.

Hauck, S. A., Margot, J.-L., Solomon, S. C., Phillips, R. J., Johnson, C. L., Lemoine, F. G., ... Zuber, M. T. (2013). The curious case of Mercury's internal structure. J. Geophys. Res., 118(6), 1204-1220. doi: 10.1002/jgre.20091

Heyner, D., Nabert, C., Liebert, E., \& Glassmeier, K.-H. ～（2016). Concerning reconnection-induction balance at the magnetopause of Mercury. J. Geophys. Res. (Spa. Phys.), 121. doi: 10.1002/2015JA021484 
Heyner, D., Wicht, J., Gómez-Pérez, N., Schmitt, D., Auster, H.-U., \& Glassmeier, K.-H. (2011). Evidence from Numerical Experiments for a Feedback Dynamo Generating Mercury's Magnetic Field. Science, 334. doi: $10.1126 /$ science. 1207290

Holme, R., \& Bloxham, J. (1996). The magnetic fields of Uranus and Neptune: Methods and models. J. Geophys. Res., 101, 2177-2200.

Hood, L., \& Schubert, G. (1979). Inhibition of solar wind impingement on Mercury by planetary induction currents. J. Geophys. Res., 84. doi: 10.1029/ JA084iA06p02641

Jackson, D. D. (1979). The use of a priori data to resolve nonuniqueness in linear inversion. Geophys. J. R. Astr. Soc., 57, 137-157.

Johnson, C. L., Philpott, L. C., Anderson, B. J., Korth, H., Hauck, S. A., Heyner, D., ... Solomon, S. C. (2016). MESSENGER observations of induced magnetic fields in Mercury's core. Geophys. Res. Lett., 43. doi: 10.1002/2015GL067370

Johnson, C. L., Purucker, M. E., Korth, H., Anderson, B. J., Winslow, R. M., Al Asad, M. M. H., ... Solomon, S. C. (2012). MESSENGER observations of Mercury's magnetic field structure. J. Geophys. Res., 117. doi: 10.1029/2012JE004217

Korth, H., Johnson, C. L., Philpott, L., Tsyganenko, N. A., \& Anderson, B. J. (2017). A Dynamic Model of Mercury's Magnetospheric Magnetic Field. Geophys. Res. Lett., 44, 10. doi: 10.1002/2017GL074699

Korth, H., Tsyganenko, N. A., Johnson, C. L., Philpott, L. C., Anderson, B. J., Al Asad, M. M., ... McNutt, R. L. (2015). Modular model for Mercury's magnetospheric magnetic field confined within the average observed magnetopause. J. Geophys. Res., 120, 4503-4518. doi: 10.1002/2015JA021022

Lall, U., \& Mann, M. (1995). The Great Salt Lake: A Barometer of Low-Frequency Climatic Variability. Water Res. Res., 31. doi: 10.1029/95WR01950

Langel, R. A. (1987). Geomagnetism, Vol. 1, Jacobs, J. A., Ed. In (p. 249-512). Academic Press.

Langlais, B., Purucker, M. E., \& Mandea, M. (2004). Crustal magnetic field of Mars. J. Geophys. Res., 109. doi: 10.1029/2003JE002048

Lesur, V., Rother, M., Wardinski, I., Schachtschneider, R., Hamoudi, M., \& Chambodut, A. (2015). Parent magnetic field models for the IGRF-12 GFZ- 
candidates. Earth Planets Space, 67. doi: 10.1186/s40623-015-0239-6

Levenberg, K. (1944). A Method for the Solution of Certain Non-Linear Problems in Least Squares. Quart. Appl. Math., 2, 164-168.

Lowes, F. J. (1966). Mean-square values on sphere of spherical harmonic vector fields. J. geophys. Res., 71, 2179.

Mann, M. E., \& Lees, J. M. (1996). Robust estimation of background noise and signal detection in climatic time series. Climate Change, 33, 409-445.

Mann, M. E., \& Park, J. (1993). Spatial correlations of interdecadal variation in global surface temperatures. Geophys. Res. Lett., 20. doi: 10.1029/93GL00752

Margot, J. L., Peale, S. J., Jurgens, R. F., Slade, M. A., \& Holin, I. V. (2007). Large Longitude Libration of Mercury Reveals a Molten Core. Science, 316. doi: 10 $.1126 /$ science.1140514

Mauersberger, P. (1956). Das Mittel der Energiedichte des geomagnetischen Hauptfeldes an der Erdoberfläche und seine säkulare Änderung. Gerlands Beiträge zur Geophysik, 65, 207-215.

Ness, N. F., Behannon, K. W., Lepping, R. P., Whang, Y. C., \& Schatten, K. H. (1974a). Magnetic Field Observations near Mercury: Preliminary Results from Mariner 10. Science, 185, 151-160.

Ness, N. F., Behannon, K. W., Lepping, R. P., Whang, Y. C., \& Schatten, K. H. (1974b). Observations at Mercury encounter by the plasma science experiment on Mariner 10. Science, 185, 159-170.

Oliveira, J. S., Langlais, B., Pais, M. A., \& Amit, H. (2015). A modified Equivalent Source Dipole method to model partially distributed magnetic field measurements, with application to Mercury. J. Geophys. Res., 120. doi: 10.1002/2014JE004734

Olsen, N. (1999). Induction studies with satellite data. Surveys in Geophysics, 20, 309-340. doi: 10.1023/A:1006611303582

Park, J., Lindberg, C. R., \& Vernon, F. L. (1987). Multitaper spectral analysis of high-frequency seismograms. J. Geophys. Res., 92, 12675-12684.

Parkinson, W. D., \& Hutton, V. R. S. (1989). The electrical conductivity of the Earth. Geomatik, 3, 261-321.

Percival, D. B., \& Walden, A. T. (1993). Spectral analysis for physical applications. 
multitaper and conventional univariate techniques. Cambridge, UK: Cambridge University Press.

Ridley, V. A., \& Holme, R. (2016). Modeling the Jovian magnetic field and its secular variation using all available magnetic field observations. J. Geophys. Res., 121. doi: 10.1002/2015JE004951

Rikitake, T. (1966). Electromagnetism and the Earth's Interior. Elsevier Publishing Company, Amsterdam, London, New York.

Rivoldini, A., \& Van Hoolst, T. (2013). The interior structure of Mercury constrained by the low-degree gravity field and the rotation of Mercury. Earth Planet. Sci. Lett., 377. doi: 10.1016/j.epsl.2013.07.021

Rivoldini, A., Van Hoolst, T., \& Verhoeven, O. (2009). The interior structure of Mercury and its core sulfur content. Icarus, 201. doi: $\{10.1016 /$ j.icarus.2008 $.12 .020\}$

Slavin, J. A., Owen, J. C. J., Connerney, J. E. P., \& Christon, S. P. (1997). Mariner 10 observations of field-aligned currents at Mercury. Planetary and Space Science, 45, 133-141. doi: 10.1016/S0032-0633(96)00104-3

Solomon, S. C., McNutt, R. L., Gold, R. E., \& Domingue, D. L. (2007). MESSENGER Mission Overview. Space Sci. Rev, 131. doi: 10.1007/s11214-007-9247-6

Stevenson, D. J. (1987). Mercury's magnetic field - A thermoelectric dynamo? Earth Planet. Sci. Lett., 82. doi: 10.1016/0012-821X(87)90111-7

Suess, S. T., \& Goldstein, B. E. (1979). Compression of the Hermean magnetosphere by the solar wind. J. Geophys. Res., 84. doi: 10.1029/JA084iA07p03306

Tarantola, A. (1987). Inverse problem theory. Methods for data fitting and model parameter estimation. Amsterdam: Elsevier, 1987.

Tarits, P., \& Grammatica, N. (2000, December). Electromagnetic induction effects by the solar quiet magnetic field at satellite altitude. Geophys. Res. Lett., 27, 4009-4012. doi: 10.1029/1999GL011249

Thébault, E., Finlay, C. C., Beggan, C., Alken, P., Aubert, J., Barrois, O., ... Zvereva, T. (2015). International geomagnetic reference field: the twelfth generation. Earth Planets Space, 67. doi: 10.1186/s40623-015-0228-9

Thébault, E., Langlais, B., Oliveira, J. S., Amit, H., \& Leclercq, L. (2018). A timeaveraged regional model of the Hermean magnetic field. Phys. Earth Planet. Int., 276. doi: 10.1016/j.pepi.2017.07.001 
Thébault, E., Schott, J. J., \& Mandea, M. (2006). Revised Spherical Cap Harmonic Analysis (R-SCHA): Validation and Properties. J. Geophys. Res., 111. doi: 10 $.1029 / 2005 J B 003836$

Thébault, E., Vervelidou, F., Lesur, V., \& Hamoudi, M. (2012). The shortcomings of the along-track satellite filtering in planetary magnetism. Geophys. J. Int., 188, 891-907. doi: 10.1111/j.1365-246X.2011.05281.x

Thomson, D. J. (1982). Spectrum estimation and harmonic analysis. IEEE Proc., $70,1055-1096$.

Torrence, C., \& Webster, P. J. (1999). Interdecadal Changes in the ENSO-Monsoon System. J. Clim., 12. doi: 10.1175/1520-0442(1999)012〈2679:ICITEM $>2.0 . C O$; 2

Tsyganenko, N. A., \& Sitnov, M. I. (2005). Modeling the dynamics of the inner magnetosphere during strong geomagnetic storms. J. Geophys. Res., 110. doi: 10.1029/2004JA010798

Uno, H., Johnson, C. L., Anderson, B. J., Korth, H., \& Solomon, S. C. (2009). Modeling Mercury's internal magnetic field with smooth inversions. Earth Planet. Sci. Lett., 285. doi: 10.1016/j.epsl.2009.02.032

Verhoeven, O., Tarits, P., Vacher, P., Rivoldini, A., \& Van Hoolst, T.

(2009). Composition and formation of Mercury: Constraints from future electrical conductivity measurements. $\quad$ Planet. Spa. Sci., 57, 296-305. doi: 10.1016/j.pss.2008.11.015

Vernon, F. L., Fletcher, J., Carroll, L., Chave, A., \& Sembera, E. (1991). Coherence of seismic body waves from local events as measured by a small-aperture array. J. Geophys. Res., 96. doi: 10.1029/91JB00193

Winslow, R. M., Anderson, B. J., Johnson, C. L., Slavin, J. A., Korth, H., Purucker, M. E., ... Solomon, S. C. (2013). Mercury's magnetopause and bow shock from MESSENGER Magnetometer observations. J. Geophys. Res., 118. doi: $10.1002 /$ jgra. 50237

Zhang, Z., \& Pommier, A. (2017). Electrical Investigation of Metal-Olivine Systems and Application to the Deep Interior of Mercury. J. Geophys. Res., 122. doi: 10.1002/2017JE005390 
Table 1. Inversion parameters and diagnostics

\begin{tabular}{lrrr}
\hline scheme & MBF_alt & MBF_mag & MBF_a-n \\
\hline number of vector triplets & 3682144 & 3333520 & 1413988 \\
rms misfit (nT) & 31.14 & 29.68 & 26.22 \\
$\lambda_{s}$ & $4.0 \times 10^{6}$ & $4.0 \times 10^{4}$ & $4.0 \times 10^{2}$ \\
\hline
\end{tabular}

Table 2. Comparison of previous and steady magnetic field models of this study based on different modeling techniques and data selection schemes.

\begin{tabular}{crrrrr}
\hline coefficients & MBF_alt & MBF_mag & MBF_a-n & Anderson et al. 2012 & Thebault et al. 2018 \\
\hline$g_{1}^{0}$ & -197.1 & -200.0 & -215.8 & -190.0 & -213.6 \\
$g_{1}^{1}$ & -2.9 & 1.1 & 0.2 & - & 0.9 \\
$h_{1}^{1}$ & 1.5 & 0.8 & 2.7 & -74.6 & 1.5 \\
$g_{2}^{0}$ & -83.2 & -80.9 & -57.0 & - & -57.7 \\
$g_{2}^{1}$ & 3.4 & -1.5 & 1.0 & - & - \\
$h_{2}^{1}$ & 0.0 & 0.2 & -1.4 & - & - \\
$g_{2}^{2}$ & -1.4 & -0.8 & -7.0 & - & - \\
$h_{2}^{2}$ & 0.4 & -0.2 & -3.3 & -22.0 & - \\
$g_{3}^{0}$ & -15.7 & -16.3 & -36.7 & - & - \\
$g_{3}^{1}$ & 1.8 & 4.1 & 2.9 & - & - \\
$g_{3}^{1}$ & 0.3 & 0.4 & 0.8 & - & - \\
$g_{3}^{2}$ & -1.5 & -1.5 & 9.2 & - & - \\
$h_{3}^{2}$ & 0.9 & 1.3 & 2.6 & - & - \\
$g_{3}^{3}$ & -1.4 & -1.5 & -2.5 & - & - \\
$h_{3}^{3}$ & 0.3 & 0.2 & 0.1 & -239.7 \\
\hline$q_{1}^{0}$ & -39.7 & -39.2 & -23.2 & - & - \\
$q_{1}^{1}$ & 0.6 & 0.2 & -0.2 & - & - \\
$s_{1}^{1}$ & 1.3 & 1.5 & 0.4 & - & - \\
\hline & & & & & - \\
\end{tabular}



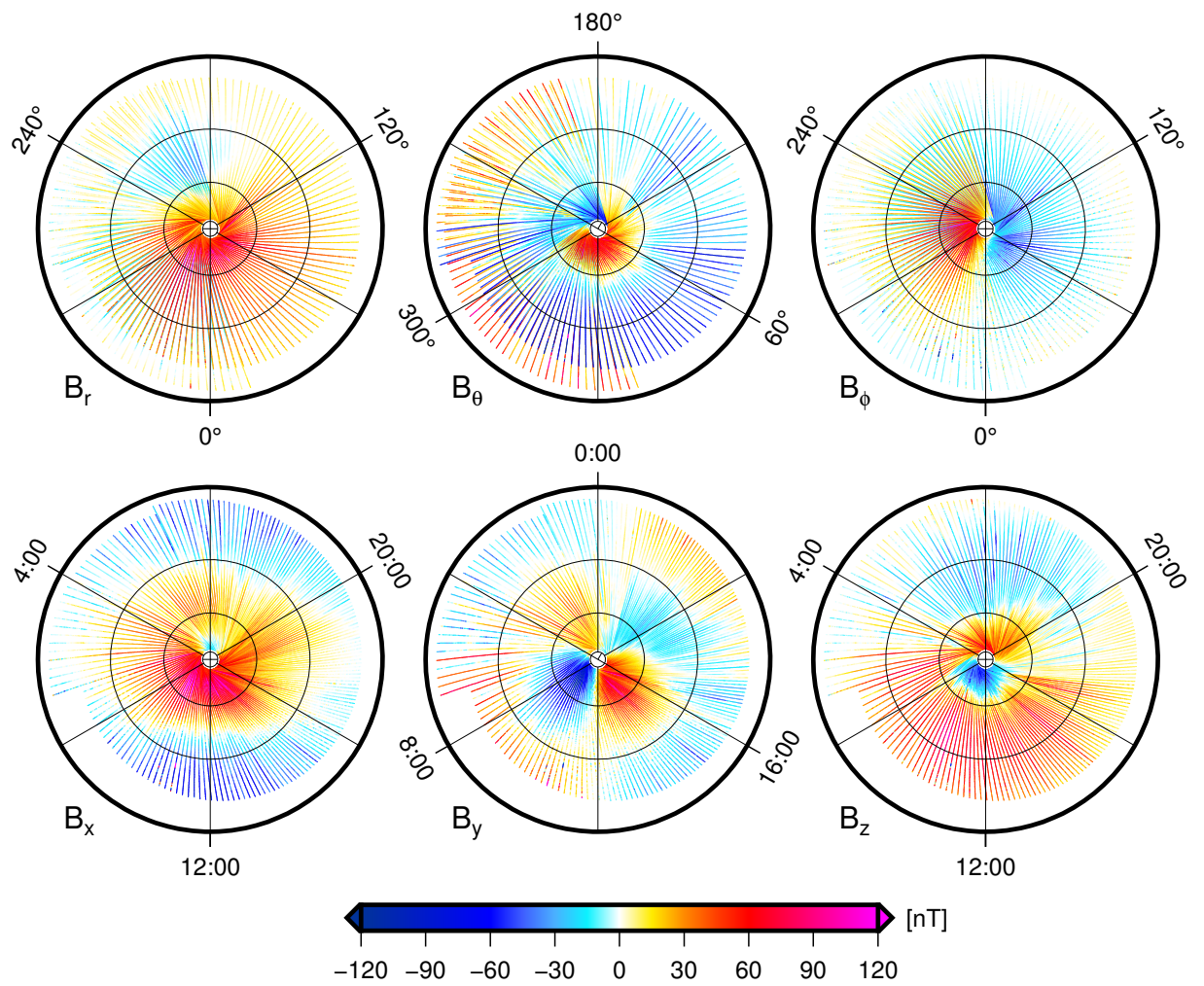

Figure 1. North polar view of residuals, after subtraction of the steady field, of the (top) $\delta B_{r}$, $\delta B_{\theta}$ and $\delta B_{\phi}$ field components in the MBF coordinate system, and (bottom) $\delta B_{x}, \delta B_{y}$ and $\delta B_{z}$ field components in the MSO coordinate system, from left to right. Maps in the bottom panel are organized with respect to local times. All maps show residuals for the period from 2011.48 to 2011.72 i.e., one Hermean year (88 terrestrial days). Black circles are spaced $30^{\circ}$ in latitude. 

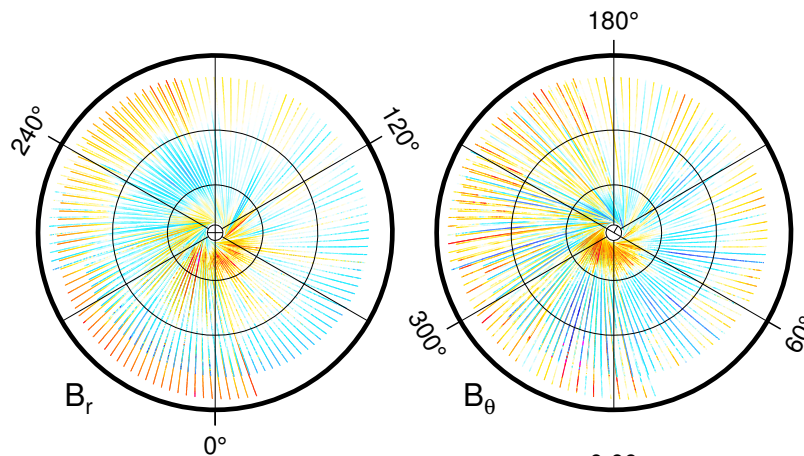

$\mathrm{B}_{\theta}$
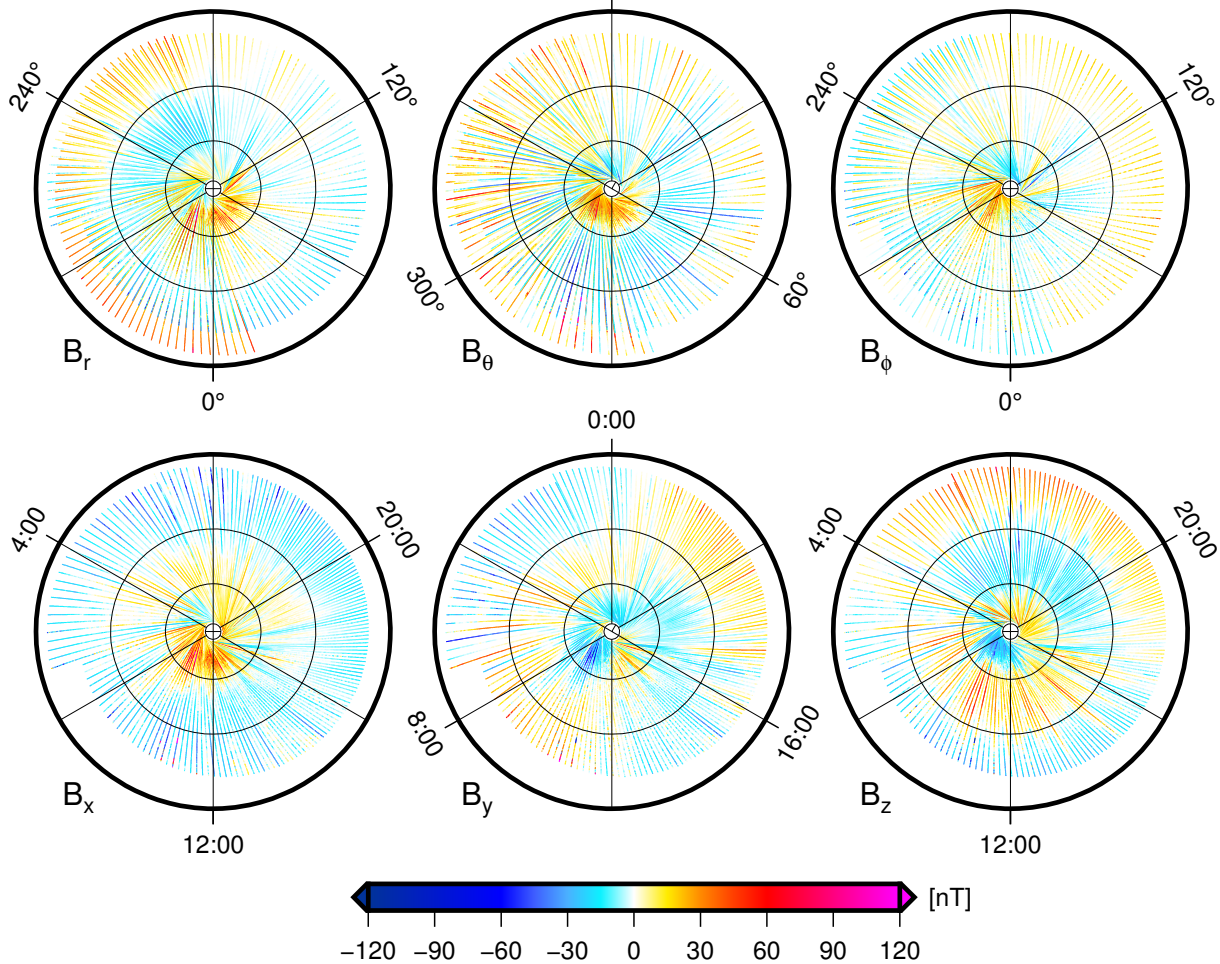

Figure 2. North polar view of residuals, after subtraction of the time-varying field. The same arrangement as in Figure 1 is applied.

Figure 3. Time series of the Gauss coefficients derived from the residual field data in the MBF coordinate system. Axial terms are shown in the top panel and equatorial terms in the bottom panel, respectively. The colors of individual curves are defined in the figure legends.

Figure 4. The temporal evolution of MESSENGER's periapsis latitude.

Figure 5. Time series of the internal and external magnetic dipole moments, $\delta M_{\text {int }}(t)$ and $M_{\text {ext }}(t)$, respectively.

Figure 6. Comparison of the time varying external magnetic dipole moment with Mercury's heliocentric distance (right axis, in astronomical units [au]). The bottom panels show the location of MESSENGER at different epochs, each when the orbiter was in a noon-midnight plane. Color depicts the altitude of the spacecraft, with the lowermost point shown as a star. The panels 1 and 2, 3 and 4, and 5 and 6 are seperated by a constant interval of 54 (terrestrial) days. 


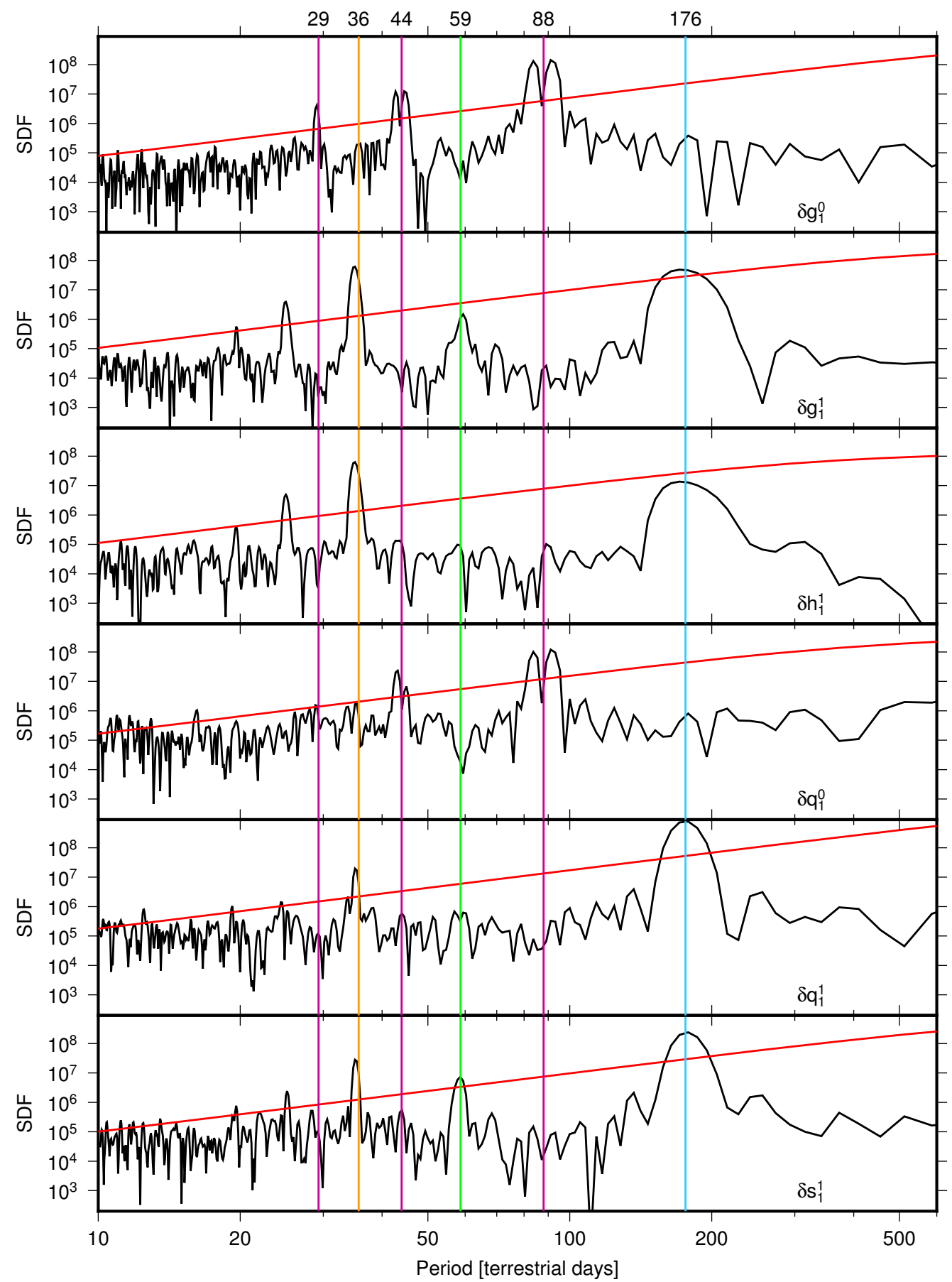

Figure 7. Power spectra of the first three internal and external field Gauss coefficients as labeled in the individual plots. Dark magenta vertical bars mark Mercury's orbital period around the Sun and its harmonics (88, 44 days and 29 days), respectively. Orange bars identify the synodic rotation period of the Sun (36 days), light-green bar marks Mercury's rotation period (59 days), and the light-blue bars mark the 176-days period, one solar day on Mercury. The red line displays $95 \%$-level of significance, corresponding to the pure line test described in the text. 


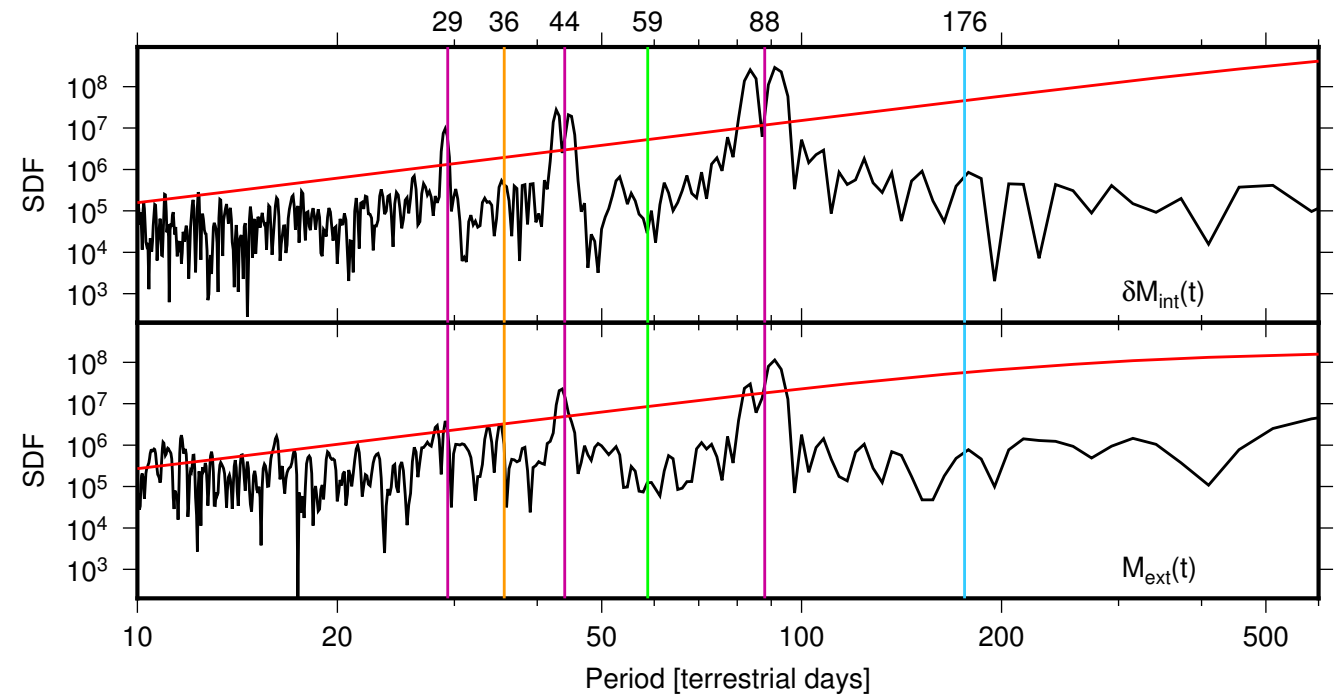

Figure 8. Power spectra of the internal and external varying dipole moments. The same line-style is applied as in Figure 7. 

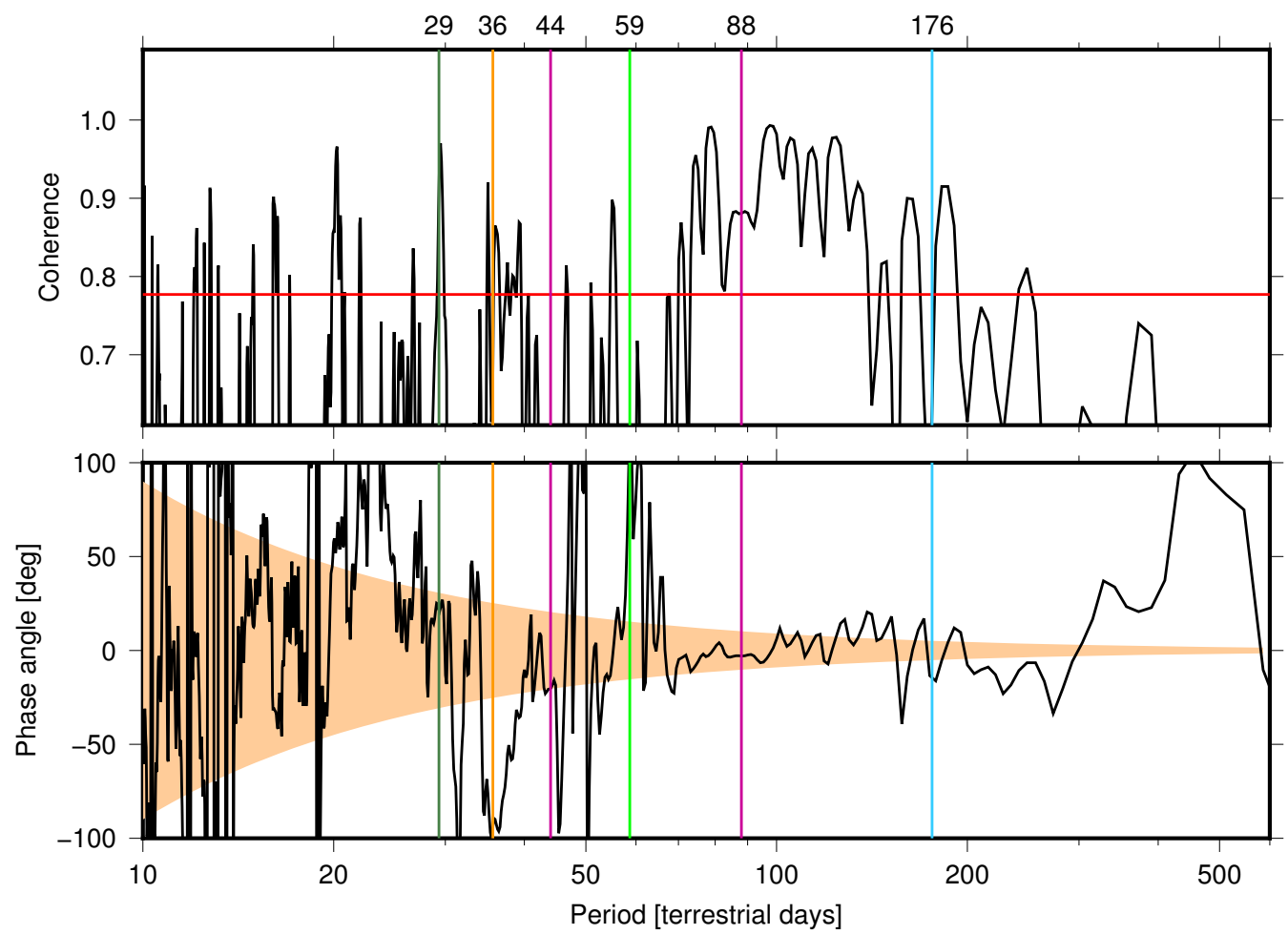

Figure 9. The coherence (top) and phase (bottom) spectra of $\delta M_{\text {int }}(t)$ and $M_{\text {ext }}(t)$ time series. The red line in the coherence spectrum marks $95 \%$ level of significance, where the colored area in the phase spectrum marks uncertainties of a 0 degree phase determined by the temporal resolution of the series. In this range phase angles are indistinguishable from zero. A positive phase angle relates to a leading of $\delta M_{\text {int }}(t)$ before $M_{\text {ext }}(t)$, whereas a negative phase angle corresponds to a trailing of $\delta M_{\text {int }}(t)$. The light colored region displays the range of un-resolved phase angles. Colors of vertical bars to identify prominent periods are the same as in Figure 8.

Figure 10. The wavelet coherence of $\delta M_{\text {int }}(t)$ and $M_{\text {ext }}(t)$. Dark red areas encircled by a gray line indicate significant coherent signal (significance level $>95 \%$ ). 
Figure A.1. Covariance matrix of the preferred model MBF_a-n. The numbering of the coefficients is $g_{1}^{0}=1 ; g_{1}^{1}=2 ; h_{1}^{1}=3 ; g_{2}^{0}=4 ; g_{2}^{1}=5$ and so on. External coefficients are 16-18.

\section{A Covariance analysis}

We study the robustness of the constrained inversion by analysing the covariance matrix that is given by

$$
\mathbf{C}=\hat{\sigma}^{2}\left(\mathbf{A}^{\top} \mathbf{C}_{\mathbf{e}}^{-1} \mathbf{A}+\mathbf{C}_{\mathbf{m}}\right)^{-1}
$$

where $\hat{\sigma}^{2}$ is the misfit of the model. The covariance matrix quantifies the uncertainties in the model estimates due to linear dependence between model parameters. Ideally, one would expect this matrix to be purely diagonal, but in fact some non-diagonal elements are not zero, which indicates a dependency between coefficients of the same degree but different order.

In Figure A.1 covariance matrix of our preferred steady field models in the MBF coordinate system is shown. The plot shows largely positive diagonal elements, which correspond to the covariance between identical coefficients, i.e. $\mathbf{C}(2,2)$ etc. We also observe large values of covariances between different coefficients. Most noticeable are large correlation between the first six internal coefficients $g_{1}^{0}, \ldots, h_{2}^{1}$. Similar structures are found for the covariances between coefficients of the first and third spherical harmonic degrees, and between degree 1 and 4 , but with smaller covariance values.

We tend to assume that large values of the covariance between different internal coefficients could be caused by the particular orbital configuration of the MESSENGER mission, with no magnetic field measurement over Mercury's southern hemisphere. Estimations of spherical harmonic coefficients with even spherical harmonic degrees may be prone to such data distribution. No significant covariance between internal and external coefficients (coefficient numbers 16,17 and 18) is found, which may indicate a good separation between these field contributions in our modeling.

Figure A.2 shows maps of covariance matrices for different epochs during the mission interval. The covariance structure of these maps varies with time, as it can be deduced from the different patterns in the maps, but values of the covariance are largely 
Figure A.2. Covariance matrices for different epochs of time varying magnetic field model derived from residuals between MBF_a-n and MESSENGER measurements. Top panel epochs around 2011.7, middle panel around 2013.2 and bottom panel around 2015.2.

reduced. Again, there seems to be no indication of significant covariance between external and internal field coefficients.

\section{B Spectral and coherence analysis}

The spectral analysis of series of the Gauss coefficients and the external and internal dipole moments is conducted by using the multi-taper method (MTM). The MTM was originally developed by Thomson (1982) and proved to provide robust spectral estimates of geophysical and climatic time series (Park et al., 1987; Percival \& Walden, 1993; Ghil et al., 2002). Furthermore, it performs particularly well for short time series (Park et al., 1987; Mann \& Lees, 1996), which may be applicable for this study. The method provides a spectral estimate with an optimal trade-off between spectral resolution and residual variance, where the trade-off is determined by the choice of the bandwidth of spectral resolution controlled by an integer $p$. The number of tapers $M$ is then defined accordingly.

We tested several MTM-parameter sets $\{p, M\}$, and the sets $\{p, M\}=\{1,1\},\{2,3\}$ show minimal variances of the residual signal between time series and their reconstructions from the spectrum. In order to have highly resolved spectra we apply $p=1, M=$ 1 in this study. Moreover, we consider the period range between 5 and 500 days as robustly resolved. The limit towards longer periods is set by the total length of time series, which is about 1492 days long and represents the duration of the mission around Mercury. This implicitly requires that periods should occur at least three times. At the short end, shorter periods than 5 days may not be resolved, because of the orbital sampling and the subsequent spline interpolation.

To estimate the coherence of two individual time series, we apply two different methods. First, we derive the coherence across two time series by following closely Vernon et al. (1991), Mann and Park (1993), and Lall and Mann (1995): the coherence of two signals is determined by the individual spectral density functions (SDF) of the series using the multi-taper method. We refer to this as MTM-coherence. Secondly, we use a wavelet 
based analysis of the series that were developed by Torrence and Webster (1999) and Grinsted et al. (2004). The wavelet-coherence provide a localized correlation coefficient in the time-frequency space (Grinsted et al., 2004), whereas the MTM-coherence gives a global (averaged) estimate. The wavelet-coherence is sensitive to quasi-periodic variations.

A coherence value of unity indicates complete dependence of one signal on another, whereas a coherence value of zero refers to no dependence of one signal on another. Two signals can only be coherent at the same frequency, and may have a phase that varies between \pm 180 degree. Both methods provide estimates of phases between coherent signals.

In this study, we apply a pure line test as given by Mann and Lees (1996) to verify the significance of spectral and coherent features against the null hypothesis of a red noise background. Contrary to a white noise process, with no correlation between single observations, red noise process include some long term correlations, such as a linear trend. The spectrum of a red noise process is estimated by the spectrum of a first order auto-regressive process. This pure line test is used to assess the robustness of our results in the next section. 